\title{
EFEITO DO TREINO ISOCINÉTICO EXCÊNTRICO SOBRE A RAZÃO I/Q DO TORQUE E EMGS EM SUJEITOS SAUDÁVEIS
}

\author{
EFFECT OF ISOKINETIC ECCENTRIC TRAINING ON H/Q TORQUE RATIO AND SEMG IN \\ HEALTHY SUBJECTS \\ EFECTO DEL ENTRENAMIENTO ISOCINÉTICO EXCÉNTRICO SOBRE EL ÍNDICE I/C DE \\ TORQUE Y EMGS EN SUJETOS SANOS
}

Artigo Original

\author{
Heleodório Honorato Santos \\ (Fisioterapeuta e Educador Físico) \\ Daniela Naomi Hanashiro 2 \\ (Fisioterapeuta) \\ Mariana Arias Ávila \\ (Fisioterapeuta) \\ Paula Rezende Camargo² \\ (Fisioterapeuta) \\ Ana Beatriz Oliveira ${ }^{2}$ \\ (Fisioterapeuta) \\ Tania Fátima Salvini \\ (Fisioterapeuta) \\ 1. Departamento de Fisioterapia, \\ Universidade Federal da Paraíba, \\ João Pessoa, PB, Brasil. \\ 2. Departamento de Fisioterapia, \\ Universidade Federal de São \\ Carlos, São Carlos, SP, Brasil.
}

\section{Correspondência:}

Av. Mons. Odilon Coutinho, 191/402 - Cabo Branco, João Pessoa, PB, Brasil, 58045-120. dorioufpb@gmail.com

\begin{abstract}
RESUMO
Objetivo: Avaliar os efeitos do treino isocinético excêntrico dos extensores do joelho sobre a razão Isquiotibiais/Quadríceps (I/Q) do torque e do eletromiograma de superfície (EMGs), em sujeitos saudáveis. Méto-

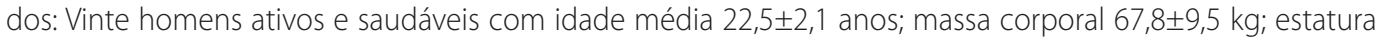
1,72 $\pm 0,10 \mathrm{~m}$; e índice de massa corporal (IMC) de $22,5 \pm 2,0 \mathrm{~kg} / \mathrm{m}^{2}$ foram avaliados quanto ao torque (isométrico e excêntrico a 30 e 120\%/s) e EMGs dos extensores e flexores do joelho, antes e após 6 semanas de treino isocinético excêntrico (30\%) dos extensores do joelho. Resultados: $O$ torque extensor do joelho aumentou em todos os modos e velocidades avaliados $(P<0,01)$; a razão I/Q do torque declinou em todas as medidas realizadas $(P<0,01)$, porém a razão I/Q do EMGs manteve-se nos valores pré-treino $(P>0,05)$. As correlações torque/EMGs mostraram-se fracas $(r<0.3 ; \mathrm{P}>0,05)$ para todos os modos de contração, no pré- e pós-treino, porém, houve diferença $(P>0,01)$ na comparação entre o modo excêntrico $\left(30^{\circ}\right.$ e $120 \%$ s) e isométrico, pré e pós-treino. Conclusões: $O$ treino isocinético excêntrico dos extensores do joelho aumentou a diferença na razão I/Q do torque, porém, não alterou a razão I/Q do EMGs, sugerindo que a adaptação pelo aumento do torque associado ao treino excêntrico não alterou o recrutamento das unidades motoras avaliadas pelo EMGs.
\end{abstract}

Palavras-chave: exercício, eletromiografia, movimento, joelho, força muscular.

\begin{abstract}
Objective: To evaluate the effects of isokinetic eccentric training of the knee extensors on Hamstring/Quadriceps (H/Q) torque and surface electromyogram (SEMG) ratio in healthy subjects. Methods: Twenty active, healthy men, with an average age of 22.5 \pm 2.1 years; body mass of $67.8 \pm 9.5 \mathrm{~kg}$; height of $1.72 \pm 0.10 \mathrm{~m}$; and body mass index (BMI) of $22.5 \pm 2.0 \mathrm{~kg} / \mathrm{m}^{2}$, were evaluated for torque (isometric and eccentric $30 \% / \mathrm{s}$ and $120 \% / \mathrm{s}$ ) and SEMG of the knee extensors and flexors, before and after 6 weeks of isokinetic eccentric training (30\%) of the knee extensors. Results: Knee extensor torque increased in all modes and speeds evaluated $(P<0.01)$, the $H / Q$ torque ratio declined in all measurements $(P<0.01)$, but the H/Q SEMG ratio remained at pre-training values $(P>0.05)$. The torque/SEMG correlation was weak $(r<0.3 ; P>0.05)$ for all modes of contraction, pre-and post-training. However, there were significant differences $(P>0.01)$ in the comparison between the eccentric (30\% and $120 \%$ s) and isometric modes, pre-and post-training. Conclusions: Isokinetic eccentric training of the knee extensors increased the difference in torque H/Q ratio, but did not alter the SEMG H/Q ratio. This suggests that adaptation by increasing the torque, associated with eccentric training, did not alter the recruitment of motor units assessed by SEMG.
\end{abstract}

Keywords: exercise, electromyography, movement, knee, muscle strength.

\section{RESUMEN}

Objetivo: Evaluar los efectos del entrenamiento isocinético excéntrico de los extensores de la rodilla sobre el indice isquiotibiales/cuadriceps (I/C) del torque y de la electromiografía de superficie (EMGs), en sujetos sanos.


tura 1,72 $\pm 0,10$ m e índice de masa corporal (IMC) de 22,5 $\pm 2,0 \mathrm{~kg} / \mathrm{m}^{2}$ fueron evaluados para el torque (isométrico y excéntrico $30^{\circ}$ y 120\%/s) y electromiografía de superficie de los extensores y de los flexores de la rodilla antes y después de 6 semanas de entrenamiento isocinético excéntrico (30\%/5) de los extensores de la rodilla. Resultados: El torque extensor de la rodilla aumentó en todos los modos y velocidades evaluados $(P<0,01)$; el índice I/C de torque se redujo en todas las mediciones $(P<0,01)$, no obstante, el índice I/C de la EMG se mantuvo en los valores del pre-entrenamiento $(P>0,05)$. Las correlaciones torque/EMG se presentaron débiles $(r<0,3, P>0,05)$ para todos los modos de contracción en las condiciones pre y post-entrenamiento, sin embargo, hubo diferencias significativas $(P>0,01)$ entre los modos excéntrico $\left(30^{\circ}\right.$ y $120 \%$ s) e isométrico en el pre y post-entrenamiento. Conclusiones: El entrenamiento isocinético excéntrico de los extensores de la rodilla aumentó la diferencia en la relación torque/IC, sin embargo, no alteró el índice I/C de la EMGs, lo que sugiere que la adaptación al aumentar el torque motor asociado con el entrenamiento excéntrico no afectó a la contratación de unidades motoras evaluadas por EMGs.

Palabras clave: ejercicio, electromiografía, movimiento, rodilla, fuerza muscular. 


\section{INTRODUÇÃO}

A dinamometria isocinética tem sido bastante utilizada na avaliação muscular, especialmente por permitir o controle de algumas variáveis, como o modo de contração, velocidade do movimento, resistência aplicada, amplitude de movimento (ADM), etc., e pela confiabilidade de seus resultados durante os testes ou treinamento de força muscular'.

O uso da eletromiografia de superfície tem sido também muito frequente para avaliar o movimento humano e no diagnóstico neuromuscular $^{2}$, onde são estudadas possíveis alterações do sinal mio-elétrico na avaliação da marcha, no uso do biofeedback em pacientes, em procedimentos terapêuticos como equilíbrio e propriocepção, e em programas de exercícios que possam influenciar diretamente o controle motor ${ }^{3}$.

A associação da dinamometria isocinética com o eletromiograma de superfície (EMGs) tem contribuído para esclarecer algumas questões sobre a função muscular, como por exemplo, comparação entre tarefas estáticas e dinâmicas ${ }^{4}$, observação do comportamento de músculos agonistas e antagonistas ${ }^{5}$, e dos efeitos de programas de treino resistido com volume fixo ${ }^{5,6}$ ou progressivo ${ }^{4}$ sobre determinada articulação ou segmento corporal.

A razão isquiotibiais/quadríceps (I/Q) do torque é uma variável muito utilizada nos estudos de dinamometria isocinética ${ }^{7}$ para observar o equilíbrio articular do joelho, quer seja na forma convencional ou funcional ${ }^{8}$. Alguns estudos mostram que esta razão varia de 40 a 80\%, dependendo da população estudada, idade e gênero, e quando é menor de 40\% há aumento da força de cisalhamento e instabilidade do joelho, sobrecarregando especialmente o ligamento cruzado anterior (LCA) 9 .

No entanto, apesar dos estudos com EMGs analisarem o comportamento do sinal frente aos vários tipos de ação muscular (isométrica, concêntrica ou excêntrica) ${ }^{4,10}$, distintas velocidades, diferentes intensidades de contração ${ }^{5,11}$, amplitudes de movimento controladas e a programas de treinamentos diversificados ${ }^{4,12}$, poucos estudos têm utilizado a razão do EMGs entre flexores e extensores do joelho como ferramenta de avaliação da função muscular ${ }^{13}$.

Apenas os estudos de Kellis e Katis ${ }^{13}$ relatam que a análise do EMGs associada ao torque fornece uma excelente ferramenta para exame da função do joelho, monitoramento de programas de reabilitação, avaliação dos efeitos de programas de treinamento, além do índice da adaptação neuromuscular ao exercício. No entanto, eles analisaram exclusivamente a razão I/Q do torque e do EMGs, sem observar se estas se alteram quando estes grupos musculares são submetidos a algum tipo de treinamento, especialmente o treino excêntrico que promove incremento da força muscular ${ }^{14}$, maior ativação neural quando comparado a outro modo de contração ${ }^{9}$, sendo considerado um importante estímulo na hipertrofia, na ativação da síntese protéica ${ }^{15}$ e na adaptação do tecido conjuntivo ${ }^{16}$.

Considerando que o treino excêntrico para fortalecimento muscular tem sido muito utilizado e que causa grande adaptação neuromuscular, o objetivo deste estudo foi avaliar o efeito do treino isocinético excêntrico para fortalecimento dos extensores do joelho sobre a razão I/Q do torque e do EMGs em sujeitos saudáveis.

\section{MATERIAL E MÉTODOS}

Foram selecionados 25 sujeitos, no entanto, cinco deles foram excluídos (três devido à dor no joelho; e dois por abandono) durante os treinos. Assim, 20 homens universitários (idade média 22,5 22,1 anos;

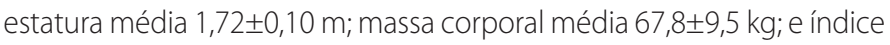
de massa corporal (IMC) média 22,5 $\pm 2,0 \mathrm{~kg} / \mathrm{m}^{2}$, ativos e saudáveis que não participavam de treino de fortalecimento muscular nem reportaram alterações ou história de lesão no joelho, concluíram o estudo. A amostra foi aleatoriamente (sistematicamente) escolhida entre os alunos do curso de graduação em Fisioterapia da Universidade Federal de São Carlos (UFSCar), São Carlos, SP, Brasil.

O estudo foi aprovado pelo Comitê de Ética da UFSCar (Parecer n 144/2004) e todos os sujeitos assinaram um Termo de Consentimento Livre e Esclarecido de acordo com a Resolução 196/96 do Conselho Nacional de Saúde com base na declaração de Helsinki. Pesquisa registrada no Australian New Zeland Clinical Trials and Registry (ANZCTR) sob nº 12607000590460.

\section{Dinamometria}

Inicialmente, foi identificada a dominância do membro inferior dos sujeitos (5 com dominância direita e 15 com dominância esquerda), perguntando qual a perna de chute, verificando qual membro se mantinha estabilizado no teste de desequilíbrio e qual membro ele apoiava para saltar um obstáculo colocado à sua frente ${ }^{12}$.

Os sujeitos se aqueceram por cinco minutos em bicicleta ergométrica (25W), na velocidade de $20 \mathrm{~km} / \mathrm{h}^{17}$. Em seguida, na posição de pé, realizaram uma série de alongamentos com três repetições (30 s de alongamento, seguidos por $30 \mathrm{~s}$ de repouso) para músculos flexores e extensores do joelho e flexores plantares, em ambos os membros ${ }^{18}$.

Após o aquecimento, os sujeitos sentaram-se no dinamômetro isocinético (Biodex Multi-Joint System 3 - Biodex Biomedical Systems, Inc., Shirley, NY), com o encosto reclinado cinco graus da posição vertical, com os joelhos flexionados a $90^{\circ}$. Para evitar movimentos compensatórios foram utilizadas faixas no tronco, pelve e região distal da coxa.

O eixo de rotação do dinamômetro foi alinhado com o eixo anatômico do joelho (epicôndilo lateral do fêmur), e o braço de alavanca foi fixado na região distal da perna ( $5 \mathrm{~cm}$ acima do maléolo medial), de forma a permitir um arco completo de dorsiflexão do tornozelo ${ }^{19}$.

Durante as avaliações e treinamento, os sujeitos receberam orientação de segurar firmemente nos apoios laterais do assento, para auxiliar na realização da força durante o movimento contra resistido. Os ajustes para correção do efeito da gravidade sobre o torque foram realizados com o joelho a $60^{\circ}$ e calculados pelo próprio software do equipamento, conforme Dvir ${ }^{19}$.

A mensuração do torque extensor e flexor do joelho foi realizada no membro não dominante (suporte), nos modos: isométrico e isocinético excêntrico (30 e 120\%). Para medida do torque isométrico máximo, o braço do dinamômetro foi fixado na posição de $60^{\circ}$ para os extensores, e de $30^{\circ}$ para os flexores ${ }^{20}$. A avaliação consistiu de uma série de três contrações isométricas voluntárias máximas (CIVM), cada uma mantida por $5 \mathrm{~s}$, com intervalo de um minuto entre elas ${ }^{4,20}$, registrando a média dos três picos de torque (PT) para cada sujeito ${ }^{15}$.

Os testes excêntricos constaram de uma série de cinco contrações excêntricas voluntárias máximas (CEVM), consecutivas, com repouso de três minutos entre os testes ${ }^{18}$ e calculadas as médias dos picos de torque (MPT) das cinco contrações, para os extensores e flexores do joelho, em cada velocidade testada (30\%/s e 120\%/s).

Antes de cada teste foram feitas familiarizações dos sujeitos no equipamento constando de três contrações excêntricas submáximas dos extensores e flexores ( $20^{\circ}$ a $90^{\circ}$ de ADM), seguidas de um minuto de repouso ${ }^{18}$.

Para geração das CEVM, foram programados, no dinamômetro isocinético, torques com 30\% acima dos valores gerados nas avaliações isométricas, valores estes que foram periodicamente aumentados durante o período de treino.

Durante as avaliações e treinamento, os sujeitos receberam estímulos verbais, pronunciados de forma padronizada ("força, força, força, mais força, mais força...") pelo mesmo avaliador para que atingissem a máxima contração, assim como feedback visual no monitor do próprio equipamento, através da visualização do gráfico ${ }^{20}$.

Para efeitos de comparação entre os sujeitos, foram considerados os 
valores dos torques, normalizados pela massa corporal, nas avaliações pré e pós-treino de cada um dos sujeitos.

A fim de permitir um tempo de recuperação suficiente da musculatura, o protocolo de treino iniciou-se $72 \mathrm{~h}$ após a avaliação inicial, e pelo mesmo motivo, a avaliação final só foi realizada considerando o mesmo tempo de recuperação a partir da última sessão de treino ${ }^{12}$.

Treinamento isocinético: Enquanto os sujeitos realizavam o protocolo de aquecimento e alongamento musculares já descritos anteriormente, eram feitas a calibração do dinamômetro (conforme manual do equipamento) e o sorteio do membro para iniciar o treinamento. Em seguida, eles executavam um programa bilateral de treinamento excêntrico para o fortalecimento dos extensores do joelho, na velocidade de $30 \%$ s, consistindo de 3 séries de 10 CEVM $^{11,19}$, com 3 minutos de repouso entre as séries ${ }^{11}$, realizadas duas vezes semanais (com intervalo mínimo de 48 horas entre as sessões), durante 6 semanas ( 12 sessões).

Eletromiografia de superfície (EMGs): Para o registro do sinal elétrico dos músculos vasto medial (VM), vasto lateral (VL), reto femoral (RF), semitendinoso (ST) e bíceps femoral (BF) ${ }^{5,21}$, foi utilizado um eletromiógrafo (modelo 800C, EMG System do Brasil) com as seguintes características técnicas: hardware com placa de conversão analógico-digital (A/D) de 12 bits; amplificador com ganho de 1000 vezes; filtro passa banda de 20 a 500 Hz (Butterworth de 2a ordem); razão de rejeição de modo comum (RRMC) >100 dB; taxa de ruído do sinal < $3 \mu \mathrm{V}$ RMS; impedância de $10^{9} \mathrm{Ohms}$; eletrodos superficiais (bipolares, ativos) com pré-amplificação de 20 vezes e um software Aqdados para coleta e análise de sinais com frequência de amostragem de $1000 \mathrm{~Hz}$, por canal, montado em plataforma Windows.

As medidas para colocação dos eletrodos foram feitas com os sujeitos em posição ortostática, o pé do membro inferior a ser avaliado sobre um apoio, mantendo quadril e joelho a $90^{\circ}$ de flexão. Para o músculo VM a 80\% da distância entre a Espinha Ilíaca Antero Superior (EIAS) e o espaço articular anterior à borda do ligamento colateral medial (LCM); para o VL a 2/3 da distância entre a EIAS e o lado lateral da patela; para o RF a 50\% da distância entre a EIAS e a borda superior da patela; para o ST a 50\% da distância entre a tuberosidade isquiática e o epicôndilo medial da tíbia; e para o BF a 50\% da distância entre a tuberosidade isquiática e o epicôndilo lateral da tíbia 22

Em seguida, a pele foi tricotomizada e limpa com álcool a 70\% para diminuir a impedância, e os eletrodos encaixados num suporte emborrachado, previamente construído nas dimensões dos eletrodos, e fixados com esparadrapo e faixas elásticas com velcro, para evitar deslizamento sobre a pele e ruído no sinal. Os eletrodos foram fixados com suas barras paralelas posicionadas perpendicularmente ao trajeto das fibras musculares 22 pelo mesmo examinador em todos os sujeitos, nas avaliações pré e pós-treino, para minimizar problemas comuns de localização de sensores apontados em estudos de EMG.

Para análise e processamento do sinal eletromiográfico, considerou-se os tempos de cinco segundos, dois segundos e meio segundo para os modos isométrico e excêntrico a 30\% s e 120\%/s, respectivamente. 0 janelamento foi realizado na porção central do sinal EMGs e calculada a média das contrações, para cada modo e velocidade avaliados.

Em seguida, os sinais foram transformados em linguagem ASCII e analisados em rotina MatLab (versão 7.1) para cálculo da Root Mean Square (RMS $[\mu V])$, que foi normalizada pela CIVM para garantir as comparações entre músculos e entre sujeitos.

\section{Análise dos dados}

Foram realizados procedimentos de estatística descritiva (média e desvio padrão) e inferenciais (teste t pareado, ANOVA One-Way e teste de correlação de Pearson) por meio do Statistical Package for the Social Sciences (SPSS) versão 15.0. Para todas as situações foi utilizado nível de significância de $5 \%(P<0,05)$.

Nas avaliações das médias dos picos de torque (MPT), da Razão I/Q do torque convencional (MPT flexores/MPT extensores X 100) e da Razão I/Q da RMS normalizada (média da RMS dos flexores/média da RMS dos extensores X 100) dos sinais eletromiográficos entre os modos e velocidades avaliadas (isométrico, excêntrico a 30\%/s e 120\%/s), utilizou-se o teste ANOVA (One-Way) seguido de um post hoc de Tukey. Nas comparações entre as médias do pré e pós-treino dessas variáveis utilizou-se o teste $t$ pareado.

O teste de Pearson foi utilizado para avaliar as possíveis correlações entre a MPT e RMS de extensores e flexores do joelho, tanto no pré quanto no pós-treino, em todos os modos e velocidades avaliados (isométrico e excêntrico a 30\%/s e 120\%/s), considerando-se a seguinte classificação: 0,0 (nula); entre 0,01 e 0,3 (fraca); entre 0,31 e 0,60 (regular); entre 0,61 e 0,90 (forte); entre 0,91 e 0,99 (muito forte); e 1 (plena ou perfeita).

\section{RESULTADOS}

A comparação da MPT dos extensores do joelho, entre pré e pós-treino (tabela 1), mostrou que houve diferença em todos os modos avaliados com aumento de 33\% para o modo excêntrico a 30\%s $(P<0,01)$ e de 15\% para os modos isométrico e excêntrico a 120\%s ( $P<0,01)$. Quanto a MPT dos flexores do joelho, não houve diferença entre pré e pós-treino em nenhum dos modos de contração avaliados $(P>0,05)$.

Quando comparados os modos de contração, houve diferença significativa na MPT para os extensores do joelho, tanto no pré $(\mathrm{P}<0,05)$ quanto no pós-treino $(P<0,001)$. No pré-treino, esta diferença foi entre o modo isométrico e excêntrico a 30\% $(P<0,01)$, e no pós-treino entre isométrico e excêntrico a 30\%/s $(P<0,001)$, e entre excêntrico a 30\%/s e 120\%/s ( $P<0,001$; tabela1).

Quanto aos flexores, houve diferença entre os modos isométrico e excêntrico a 30\% $(P<0,05)$ tanto no pré quanto no pós-treino. Vale salientar que os maiores valores de MPT ocorreram primeiro no modo excêntrico a 30\% s; seguido pelo excêntrico a 120\%/s; e finalmente pelo isométrico, tanto para extensores quanto para flexores do joelho (tabela 1).

Houve diminuição significativa na razão I/Q após o treino $(P<0,01)$, em todos os modos e velocidades avaliados (tabela 2). O maior declínio (20\%) ocorreu no modo e na velocidade de treino (excêntrico a 30\%), seguido do modo excêntrico a 120\% (13\%) e do modo isométrico (10\%).

No pré-treino, houve diferença entre os modos isométrico e excêntrico a 120\%/s ( $P<0,05)$, enquanto que no pós-treino houve diferença entre os modos isométrico e excêntrico a 30\%s $(P<0,05)$, mas principalmente, entre os modos excêntricos a 30\%/s e 120\%s $(P<0,01)$.

A comparação dos valores da RMS entre pré e pós-treino (tabela 3) aumentou, tanto para os extensores (isométrico: 8,5\%; $\mathrm{P}<0,05$; excêntrico a 30\%s: 28,2\%; e 120\%/s: 20,5\%; P<0,01), quanto para os flexores do joelho

Tabela 1. Médias dos picos de torque (MPT) dos extensores e flexores do joelho normalizadas pela massa corporal, pré e pós-treino excêntrico dos extensores.

\begin{tabular}{c|c|c|c|c|c|c}
\hline \multirow{2}{*}{$\begin{array}{c}\text { Modos de } \\
\text { contração }\end{array}$} & \multicolumn{3}{|c|}{ Extensores (Nm) } & \multicolumn{3}{c}{ Flexores (Nm) } \\
\cline { 2 - 7 } & Pré-treino & Pós-treino & $\begin{array}{c}\text { Teste } t \\
\text { (pareado) }\end{array}$ & Pré-treino & Pós-treino & $\begin{array}{c}\text { Teste } t \\
\text { (pareado) }\end{array}$ \\
\hline Isométrico & $324,0 \pm 55,6$ & $383,4 \pm 54,3$ & $<0,01$ & $160,1 \pm 38,1$ & $168,8 \pm 40,4$ & 0,07 \\
\hline $\begin{array}{c}\text { Excêntrico } \\
30 \% / 5\end{array}$ & $387,3 \pm 78,0^{*}$ & $502,0 \pm 55,8^{* *}$ & $<0,01$ & $192,5 \pm 37,9^{*}$ & $200,5 \pm 32,9^{*}$ & 0,13 \\
\hline $\begin{array}{c}\text { Excêntrico } \\
120 \% / 5\end{array}$ & $344,0 \pm 67,6$ & $406,6 \pm 75,4$ & $<0,01$ & $186,2 \pm 37,3$ & $188,6 \pm 37,9$ & 0,67 \\
\hline
\end{tabular}

Teste ANOVA (one-way) * $\mathrm{P}<0,05$, comparado à contraçăo isométrica (pré e pós-treino); ** $\mathrm{P}<0,01$, comparado à contração isométrica e excêntrica a 120\%/s (pós-treino). 
Tabela 2. Razão isquiotibiais/quadríceps do torque pré e pós-treino isocinético excêntrico dos extensores do joelho.

\begin{tabular}{c|c|c|c|c}
\hline \multirow{2}{*}{$\begin{array}{c}\text { Modos de } \\
\text { contração }\end{array}$} & Pré-treino & Pós-treino & $\begin{array}{r}\text { Teste } t \\
\text { (pareado) }\end{array}$ & Diferença (\%) \\
\cline { 2 - 5 } & $49,8 \pm 8,3$ & $45,1 \pm 7,8$ & $<0,01$ & 10 \\
\hline Isométrico & $50,4 \pm 7,5$ & $40,3 \pm 7,0^{* *}$ & $<0,01$ & 20 \\
\hline $\begin{array}{c}\text { Excêntrico 30\%/s } \\
\text { Excêntrico } \\
120 \% / s\end{array}$ & $54,8 \pm 10,3^{*}$ & $47,7 \pm 12,3$ & $<0,01$ & 13 \\
\hline
\end{tabular}

Teste ANOVA (one-way) * $\mathrm{P}<0,05$, comparado à razão $\mathrm{I} / \mathrm{Q}$ isométrica (pré-treino); ** $\mathrm{P}<0,01$, comparado à razões I/Q isométrica e excêntrica a 120\% (pós-treino):

Tabela 3. Root Mean Square (RMS) do EMG de superfície dos músculos extensores e flexores do joelho pré e pós-treino isocinético excêntrico dos extensores do joelho, normalizada pela CIVM

\begin{tabular}{c|c|c|c}
\hline $\begin{array}{c}\text { Modos de } \\
\text { contração }\end{array}$ & Pré-treino & Pós-treino & Valor $\mathbf{P}$ \\
\hline Extensores & & & \\
\hline Isométrico & $1,32 \pm 0,05$ & $1,30 \pm 0,04$ & $0,040 \diamond$ \\
\hline Excêntrico a 30\%/s & $0,84 \pm 0,19 \neq$ & $1,17 \pm 0,36 \neq$ & $0,001 \diamond$ \\
\hline Excêntrico a 120\%/s & $0,93 \pm 0,19 \neq$ & $1,17 \pm 0,35 \neq$ & $0,003 *$ \\
\hline Flexores & & & \\
\hline Isométrico & $1,29 \pm 0,04$ & $1,28 \pm 0,03$ & $0,040 \diamond$ \\
\hline Excêntrico a 30\%/s & $0,98 \pm 0,23 \neq$ & $1,30 \pm 0,51$ & $0,007 \diamond$ \\
\hline & $1,11 \pm 0,27 \dagger$ & $1,25 \pm 0,38$ & 0,104 \\
\hline
\end{tabular}

Comparaçăo pré e pós-treino: * $\mathrm{P}<0,01$ (teste t pareado); $\oslash \mathrm{P}<0,05$ (teste de Wilcoxon); Teste ANOVA (one-way): $+\mathrm{P}<0,05$ e $\neq \mathrm{P}<0,01$ comparado ao modo isométrico (pré e pós-treino).

(isométrico: 8,5\%; $\mathrm{P}<0,05$; excêntrico a 30\%/s: 24,6\%; $\mathrm{P}<0,01$ ). No entanto, não houve diferença no modo excêntrico a 120\%s (11,0\%; P>0,05).

A análise comparativa entre os modos de contração no movimento de extensão do joelho mostrou que a RMS foi menor nos modos excêntricos a 30\% s e 120\% s quando comparado ao modo isométrico $(\mathrm{P}<0,01)$, tanto no pré quanto no pós-treino. Quanto aos flexores do joelho, houve diminuição significativa na RMS apenas no pré-treino, para o modo excêntrico a 30\%/s e 120\%/s ( $P<0,01$; $P<0,05$, respectivamente), quando comparado ao isométrico.

De modo geral, pode-se observar que as correlações entre MPT e RMS foram fracas $(r<0,3 ; P>0,05)$, para extensores e flexores do joelho, tanto no pré quanto no pós-treino, em todos os modos de contração avaliados. As correlações foram também negativas no modo excêntrico, para ambas as velocidades, e positivas para o modo isométrico (Tabela 4).

A comparação da razão I/Q do EMGs (Tabela 5) de flexores e extensores do joelho mostrou que não houve diferença entre os valores pré e pós-treino para nenhum dos modos de contração $(P>0,05)$. No entanto, houve diferença na comparação dos valores entre os modos de contração (excêntrico a 30\% e 120\%/s em relação ao modo isométrico; $\mathrm{P}<0,01)$, tanto no pré quanto no pós-treino.

\section{DISCUSSÃO}

O aumento do torque identificado no pós-treino dos extensores do joelho no presente estudo corrobora com resultados de outros autores que também realizaram trabalhos com treino excêntrico ${ }^{12,23,24}$, mostrando que este modo de contração favorece o ganho de torque em comparação aos demais (isométrico ou concêntrico). O maior ganho de torque no modo e velocidade do treinamento também já foi descrito em estudos
Tabela 4. Correlação entre as médias do pico de torque e EMG de superfície dos extensores e flexores do joelho, pré e pós-treino isocinético excêntrico dos extensores do joelho.

\begin{tabular}{|c|c|c|c|c|}
\hline $\begin{array}{l}\text { Modos de } \\
\text { contração }\end{array}$ & Pré-treino ( $r$ ) & Valor P & Pós-treino ( $r$ ) & Valor $\mathrm{P}$ \\
\hline \multicolumn{5}{|l|}{ Extensores } \\
\hline $\begin{array}{l}\text { MPT_versus_ } \\
\text { EMG_Isom }\end{array}$ & 0,024 & $0,919^{*}$ & 0,102 & $0,667 \diamond$ \\
\hline $\begin{array}{l}\text { MPT_versus_ } \\
\text { EMG_Exc_30\% }\end{array}$ & $-0,205$ & $0,386^{*}$ & $-0,126$ & $0,595^{*}$ \\
\hline $\begin{array}{l}\text { MPT_versus_ } \\
\text { EMG_Exc_120\% }\end{array}$ & $-0,211$ & $0,372 \diamond$ & 0,221 & $0,350 \diamond$ \\
\hline \multicolumn{5}{|l|}{ Extensores } \\
\hline $\begin{array}{l}\text { MPT_versus_ } \\
\text { EMG_Isom }\end{array}$ & 0,064 & 0,7900 & $-0,260$ & $0,268^{*}$ \\
\hline $\begin{array}{l}\text { MPT_versus_- } \\
\text { EMG_Exc_30\% }\end{array}$ & $-0,074$ & $0,757 \diamond$ & $-0,233$ & $0,323 \diamond$ \\
\hline $\begin{array}{c}\text { MPT_versus_ } \\
\text { EMG_Exc_120\%s }\end{array}$ & $-0,205$ & $0,386 \diamond$ & $-0,183$ & $0,441 \diamond$ \\
\hline
\end{tabular}

r: índice de correlação. *teste de Spearman; $\diamond=$ teste de Pearson.

Tabela 5. Comparação da razão I/Q do EMG de superfície pré e pós-treino excêntrico em cada modo de contração.

\begin{tabular}{c|c|c|c}
\hline \multirow{2}{*}{$\begin{array}{c}\text { Modos de } \\
\text { contração }\end{array}$} & \multicolumn{3}{|c}{ Razão I/Q do EMG (\%) } \\
\cline { 2 - 4 } & Pré-treino & Pós-treino & Teste $t$ (pareado) \\
\hline Isométrico & $0,98 \pm 0,04$ & $1,11 \pm 0,03$ & 0,741 \\
\hline Excêntrico a 30\%/s & $120,21 \pm 28,27 \neq$ & $115,62 \pm 45,96 \neq$ & 0,502 \\
\hline Excêntrico a 120\% & $121,88 \pm 30,28 \neq$ & $111,82 \pm 32,49 \neq$ & 0,286 \\
\hline
\end{tabular}

Comparação pré e pós-treino: *P<0,01 (teste t pareado); $\pm \mathrm{P}<0,05$ (teste de Wilcoxon); Teste ANOVA (one-way): ₹ $\mathrm{P}<0,01$ comparado ao modo isométrico (pré e pós-treino).

anteriores ${ }^{6,11}$. O programa de treino utilizado também favoreceu um aumento de 15\% para os modos isométrico e excêntrico a 120\%s, similar ao já evidenciado anteriormente por Coburn et al..$^{25}$ quando confrontaram outros modos de treinamento com o exercício excêntrico.

Alguns estudos têm mostrado que o remodelamento neural parece ter uma maior contribuição no ganho de força nas oito primeiras semanas de treino resistido ${ }^{23}$ do que a hipertrofia, caracterizada pelo aumento da área de secção transversa do músculo ${ }^{12}$. Como o programa de treino aplicado neste estudo foi de seis semanas, provavelmente os ganhos de torque observados sejam, preferencialmente, devidos às adaptações neurais.

Nossos resultados mostraram que o programa de treino utilizado promoveu uma diminuição importante (20\%) na razão I/Q convencional do torque, especialmente na velocidade treinada, onde a taxa dos flexores representou 40\% dos extensores. Também, no modo excêntrico a 120\%/s (13\%) e isométrico (10\%), houve diminuição desta razão, ocasionada pelo significativo aumento no torque dos extensores com o treino. Conforme Kellis e Baltzopoulus ${ }^{26}$ e Rosene et al. ${ }^{7}$, quando a razão I/Q do torque atinge valores $\leq 30 \%$ é um dos fatores preditivos de lesão do LCA, ocasionada pela redução da coativação dos flexores durante a extensão do joelho com descarga de peso.

Dessa forma, para não atingir patamares que poderiam contribuir para a desestabilização articular (razão I/Q<40\%), o programa de treinamento aplicado no presente estudo foi realizado em curto período (seis semanas), porém suficiente para induzir uma adaptação sem grandes 
riscos articulares. Essa precaução foi baseada num estudo de LaStayo et al. ${ }^{9}$ sobre a contribuição da contração excêntrica para lesão, prevenção, reabilitação e esporte, no qual eles afirmam que quando o exercício excêntrico é excessivamente empregado, predominantemente num grupo muscular, este pode levar à instabilidade articular.

Em nosso estudo, apesar dos valores da RMS do EMGs terem aumentado com o treino, eles foram menores nos modos excêntricos quando comparados ao isométrico. Estudos prévios têm demonstrado que alterações neurais induzidas pelo treinamento dependem das características do programa utilizado (intensidade, duração, freqüência, etc.), especialmente do modo de treino (isométrico, concêntrico ou excêntrico) ${ }^{27}$.

Guilhem et al. ${ }^{21}$, que além de outras variáveis (torque, velocidade angular e arquitetura muscular), compararam a atividade da RMS do EMGs de extensores (VM, VL e RF) do joelho, na contração excêntrica isocinética versus isotônica, observaram uma menor amplitude do sinal eletromiográfico associada ao exercício isocinético. Embora estes achados se coadunem, em parte, com nossos resultados, eles não avaliaram o efeito do treino, nem fizeram a comparação entre contração excêntrica e isométrica.

Os menores valores na amplitude do sinal EMGs (RMS) observados no modo excêntrico (30 e 120\%s), quando comparado ao isométrico no presente estudo, também podem estar associados às alterações no tecido conjuntivo promovido pelo treino excêntrico. Sabe-se que em resposta à ação excêntrica a membrana extracelular aumenta a expressão, síntese e acúmulo de colágeno no músculo, fortalecendo os elementos passivos que contribuem na geração de força ${ }^{16}$, necessitando, portanto, de menor recrutamento de unidades motoras (UMs) para execução da tarefa ${ }^{28}$.

Algumas hipóteses têm sido consideradas para explicar a menor amplitude do EMGs (RMS) na contração excêntrica, tais como: a ocorrência de contínuos desligamentos das pontes cruzadas antes da liberação de ATP, requerendo, portanto, mais força durante ações excêntricas com um menor consumo de oxigênio; dano ao sistema excitação- contração ${ }^{29}$; padrão dessincronizado da atividade eletromiográfica quando o exercício excêntrico é realizado por sujeitos destreinados ${ }^{16}$; mecanismo de inibição neural que consiste da ativação de vias centrais (descendentes) e periféricas (órgão tendinoso de Golgi, fuso muscular e receptores articulares) visando proteger as estruturas articulares de lesão, quando são produzidas grandes forças ${ }^{28,30}$.

Por outro lado, Suzuki et al..$^{31}$ observaram que altos níveis de força estão associados ao aumento da atividade eletromiográfica. Assim, mudanças no sinal eletromiográfico são usadas para inferir alterações da atividade neural associada à contração muscular. Nesse sentido, o estudo de Norrbrand et al. ${ }^{24}$, que analisou o efeito do treino excêntrico comparado ao concêntrico, no quadríceps (VM e VL), observou após cinco semanas de treino ( 12 sessões / 4 séries $X 7$ RM cada) aumento significativo na RMS dos extensores do joelho $(P<0,05)$ no grupo que realizou treino excêntrico, diferindo, portanto, dos nossos resultados, embora o treino adotado por eles não tenha sido isocinético.

Como proposto por Mullany et al. ${ }^{30}$, grandes forças geradas pelo quadríceps podem promover mudanças na coativação dos músculos isquiotibiais devido à uma rede neural supra-espinhal comum aos dois grupos musculares, recrutando um pool de motoneurônios agonistas e antagonistas, o que possivelmente explicaria o aumento significativo na RMS no modo isométrico $(P<0,05)$ e excêntrico a $30 \%$ s $(p<0,01)$ no pós-treino dos músculos flexores do joelho.

Por outro lado, o estudo de Remaud et al. ${ }^{32}$, comparando oito semanas de treino isotônico e isocinético com estudantes saudáveis do sexo masculino, mostrou que apesar da atividade eletromiográfica do quadríceps ter aumentado nos dois modos de contração, a co-ativação dos isquiotibiais permaneceu inalterada.
Corroborando, ainda, nossos resultados, onde a maior amplitude RMS foi no modo isométrico, tanto para extensores quanto para flexores no pré e pós-treino, Seger et al. ${ }^{6}$ relatam que durante a ação isométrica, onde apenas os elementos contráteis são ativados, a necessidade de maior quantidade de UMs ativas é diretamente proporcional à força gerada, uma vez que não há participação de componentes passivos do músculo.

Similarmente ao nosso estudo, no qual foi observada uma maior atividade EMG no modo isométrico, seguido pelo modo excêntrico a 120 e 30\%s, Kellis e Baltzopoulos ${ }^{26}$ também observaram que a ativação neural (EMG) resultante de testes isocinéticos é dependente do modo de contração e da velocidade angular, mostrando que em maiores velocidades as ações excêntricas estão associadas à maior atividade EMG, quando comparadas a velocidades menores. No entanto, Miller e Croce $^{33}$ mostraram que a amplitude do EMG foi inversamente dependente da velocidade do movimento em testes isocinéticos observando que, com o aumento da velocidade, a RMS do sinal eletromiográfico diminuiu em todas as velocidades testadas (100, 200, 300 e 400\%/s) durante a extensão do joelho.

De modo geral, há resultados conflitantes em relação à atividade eletromiográfica nos diversos modos de contração. Alguns autores relatam menor amplitude do sinal EMGs nas ações excêntricas quando comparadas às isométricas ou concêntricas ${ }^{17,33}$, enquanto que outros mostram aumento na amplitude do sinal ${ }^{10,11}$.

Em relação à correlação torque/EMG, nossos resultados mostram que, apesar dos aumentos significativos no torque, em todos os modos e velocidades avaliados as correlações foram fracas $(r<0.3 ; P>0,05)$, sugerindo que os mecanismos de ativação podem ser diferentes. Estes resultados podem ser explicados pelo fato de que a razão I/Q do torque mostrou diferenças altamente significativas entre valores pré e pós-treino $(P<0,01)$, enquanto que a razão $\mathrm{I} / \mathrm{Q}$ do EMGs não apresentou diferença $(P>0,05)$ entre estes valores, indicando que o aumento do sinal eletromiográfico foi distinto do aumento do torque.

Nossos resultados são similares aos de McCarthy, Pozniak, e Agre ${ }^{34}$ e Rabita, Pérot, e Lensen-Corbiel ${ }^{35}$, que também mostraram baixos e negativos índices de correlação torque/EMG, indicando grande variabilidade individual no grupo treinado nos modos e velocidades estudados, tanto no pré quanto no pós-treino. Por outro lado, Judge, Moreau, Burke ${ }^{4}$ e Andersen et al. ${ }^{10}$, encontraram moderadas ( $r=0.63$; $P<0.01)$ e fortes $(r=0.77 ; P<0.01)$ correlações torque/EMG, respectivamente, sugerindo que ocorre uma melhora na adaptação neural dos extensores do joelho após aplicação de um programa de treino resistido. Entretanto, esses estudos utilizaram programas com exercícios isotônicos, concêntricos e excêntricos, com resistência progressiva num período de 4 e 6 meses em atletas e ativos saudáveis, respectivamente, diferindo em modo, volume e duração do programa de treino aplicado em nosso estudo.

Na revisão da literatura científica, foi observada ausência de estudos avaliassem o efeito de um programa de treino isocinético na razão I/Q do EMGs. Há apenas estudos que comparam resultados entre membros com e sem lesão do LCA, em diferentes ângulos de movimento do joelho e como se comporta esta variável entre homens e mulheres durante o agachamento unipodal ${ }^{13}$.

A grande variabilidade nos parâmetros de treino e testes utilizados nos estudos já realizados ${ }^{10,11,17}$ dificultam uma discussão mais profunda dos nossos resultados. Porém, o treino isocinético utilizado no nosso estudo possibilita um maior controle de algumas variáveis (velocidade de contração, ADM, modo de contração, etc.) e permite analisar o sinal EMGs de modo sincronizado à avaliação do torque articular.

O presente estudo apresenta algumas limitações, tais como: 1) por razões técnicas, não foram feitas as avaliações concêntricas a 30\%s e 
$120 \%$ s, que favoreceriam a discussão, visto que a maioria dos estudos faz comparações entre o modo concêntrico e excêntrico; e 2) não ter avaliado o torque passivo que permitiria uma análise melhor da adaptação dos tecidos não contráteis e suas possíveis implicações com o sinal EMGs.

\section{CONCLUSÃO}

O treino isocinético excêntrico dos extensores do joelho aumentou a diferença na razão I/Q do torque, porém, não alterou a razão I/Q do EMGs, sugerindo que a adaptação pelo aumento do torque associado ao treino excêntrico não alterou o recrutamento das unidades motoras avaliadas pelo EMGs.

\section{AGRADECIMENTOS}

Os autores agradecem aos estudantes do curso de Fisioterapia da UFSCar, pela participação no estudo; à Fundação de Amparo à Pesquisa do Estado de São Paulo (FAPESP), e Conselho Nacional de Desenvolvimento Científico e Tecnológico (CNPq). Heleodório Honorato dos Santos foi bolsista de doutorado da Coordenação de Aperfeiçoamento de Pessoal de Nível Superior (CAPES).

Todos os autores declararam não haver qualquer potencial conflito de interesses referente a este artigo.

\section{REFERÊNCIAS}

1. Von Porat A, Henriksson M, Holmström E, Roos EM. Knee kinematics and kinetics in former soccer players with a 16-year-old ACL injury - the effects of twelve weeks of knee-specific training. BMC Musculoskelet Disord 2007;8(35).

2. Kleissen RFM, Buurke JH, Harlaar J, Zilvoid G. Electromyography in the biomechanical analysis of human movement and it clinical application. Gait Post. 1998;8:143-58.

3. Stegeman DF, Blok JH, Hermes HJ, Roeleveld K. Surface EMG models: properties and applications. J Electromyogr Kinesiol. 2000;10:313-26.

4. Judge LW, Moreau C, Burke JR. Neural adaptations with sport-specific resistance training in highly skilled athletes. J Sports Sci. 2003;21:419-27.

5. Shields RK, Madhavan S, Gregg E, Leitch J, Petersen B, Salata S, Wallerich S. Neuromuscular control of the knee during a resisted single-limb squat exercise. Am J Sports Med. 2005;33(10):1520-6.

6. Seger JY, Arvidsson B, Thorstensson A. Specific effects of eccentric and concentric training on muscle strength and morphology in humans. Eur J Appl Physiol. 1998;79:49-57.

7. Rosene JM, Forgarty TD, Mahaffey BL. Isokinetic hamstrings: quadriceps ratios in intercollegiate athletes. J Athl Train. 2001;36(4):378-83

8. Tourny-Chollet C, Leroy D. Conventional vs. dynamic hamstring-quadriceps strength ratios: a comparison between players and sedentary subjects. Isokinetics Exerc Sci. 2002;10:183-92.

9. LaStayo PC, Woolf JM, Lewek MD, Snyder-Mackler L, Reich T, Lindstedt SL. Eccentric muscle contractions: their contribution to injury, prevention, rehabilitation and sport. J Orthop Sports Ther. 2003;33:557-71

10. Andersen LL, Andersen $\mathrm{J}$, Magnusson SP, Aagaard P. Neuromuscular adaptations to detraining following resistance training in previously untrained subjects. Eur J Appl Physiol. 2005:93:511-8.

11. Higbie EJ, Cureton GL, Warren III GL, Prior BM. Effects of concentric and eccentric training on muscle strength, cross-sectional area, and neural activation. J Appl Physiol. 1996;81(5):2173-81.

12. Knight CA, Kamen G. Adaptations in muscular activation of the knee extensor muscles with strength training in young and older adults. J Electromyogr Kinesiol. 2001;11:405-12.

13. Kellis E, Katis A. Quantification of functional knee flexor to extensor moment ratio using isokinetics and electromyography. J Atlh Train. 2007;42(4):477-85.

14. Seger JY, Thorstensson A. Effects of eccentric versus concentric training on thigh muscle strength and EMG. Int J Sports Med. 2005; 26(1):45-52

15. Goldspink G. Molecular mechanisms involved in the determination of muscle fiber mass and phenotype. Adv Exerc Sports Physiol. 1999;5(2):27-39.

16. Kjær M. Role of extracellular matrix in adaptation of tendon and skeletal muscle to mechanical loading Physiol Rev. 2004;84(2):649-98.

17. Linnamo V, Strojnik V, Komi PV. EMG power spectrum and features of the superimposed M-wave during voluntary eccentric and concentric actions at different activation levels. Eur J Appl Physiol. 2002;86:534-40.

18. Coury HJCG, Brasileiro JS, Salvini TF, Poletto PR, Carnaz L, Hansson G.-A. Change in knee kinematics

during gait after eccentric isokinetic training for quadriceps in subjetcs submitted to anterior cruciate ligament reconstruction. Gait Post. 2006;24:370-4

19. Dvir Z. Isokinetics: muscle testing. Interpretation and clinical applications. 2nd ed. Edinburgh: Churchill Livingstone, 2004.

20. Pincivero DM, Salfetnikov Y, Campy RM, Coelho AJ. Angle- and gender-specific quadriceps femoris muscle recruitment and knee extensor torque. J Biomech. 2004; 37:1689-97.

21. Guilhem G, Cornu C, Guével A. Muscle architecture and EMG activity changes during isotonic and isokinetic eccentric exercises. Eur J Appl Physiol. 2011;111(11):2723-33.

22. Hermens HJ, Freriks B, Disselhorst-Klug C, Rau G. Development of recommendations for SEMG sensors and sensor placement procedures. J Electromyogr Kinesiol. 2000;40(10):361-74.

23. Carroll TJ, Stephan R, Carson RG. Neural adaptations to resistance training: implication for movement control. Sports Med. 2001;31(12):829-40.

24. Norrbrand L, Pozzo M, Tesch Per A. Flywheel resistance training calls for greater eccentric muscle activation than weight training. Eur J Appl Physiol, 2010;110:997-1005.

25. Coburn JW, Housh TJ, Malek MH, Weir JP, Cramer JT, Beck TW, Johnson GO. Neuromuscular response to three days of velocity-specific isokinetic training. J Strength Cond Res. 2006;20(4):892-8.

26. Kellis, E, Baltzopoulus $V$. The effects of antagonist muscle force on intersegmental loading during isokinetic efforts of the knee extensors. J Biomech. 1999;32:19-25.

27. Gabriel DA, Kamen G, Frost G. Neural adaptations to resistive exercise: mechanisms and recommendations for training practices. Sports Med. 2006;36:133-49.

28. Aagaard P, Simonsen EB, Andersen JL, Magnusson SP, Halkjaer-Kristensen J, Dyhre-Poulsen P. Neura inhibition during maximal eccentric and concentric quadriceps contraction: effects of resistance training. J Appl Physiol.2000; 89:2249-57.

29. Proske U, Morgan DL. Muscle damage from eccentric exercise: mechanism, mechanical signs, adaptation and clinical applications. J Physiol. 2001; 573(2):333-45.

30. Mullany H, O'Malley M, St Clair Gibson A, Vaughan C. Agonist-antagonist common drive during fatiguing knee extension efforts using surface electromyography. J Electromyogr Kinesiol. 2002;12:375-84.

31. Suzuki H, Conwit RA, Stashuk D, Santarsiero L, Metter EJ. Relationships between surface-detected EMG signals and motor unit activation. Med Sci Sports Exerc. 2002;34(9):1509-17.

32. Remaud A, Cornu C, Guével A. Neuromuscular adaptations to 8-week strength training: isotonic versus isokinetic mode. Eur J Appl Physiol. 2010;108:59-69.

33. Miller.JP, Croce RV. Effect of movement velocity and movement pattern on the Root Mean Square and the median frequency of the electromyographic activity of the quadriceps during isokinetic testing. Isokinetics Exer Sci. 2002;10:193-8.

34. McCarthy JP, Pozniak MA, Agre JC. Neuromuscular adaptations to concurrent strength and endurance training. Med Sci Sports Exerc. 2002;34(3):511-9.

35. Rabita G, Pérot C, Lensel-Corbeil G. Differential effect of knee extension isometric training on the different muscles of the quadriceps femoris in humans. Eur J Appl Physiol. 2000; 83:531-8. 